\title{
Bufadienolides induce p53-mediated apoptosis in esophageal squamous cell carcinoma cells in vitro and in vivo
}

\author{
SHAOHUAN LIN ${ }^{1}$, JUNHONG LV ${ }^{1}$, PANLI PENG ${ }^{2}$, CHANGQING CAI $^{2}$, JIANMING DENG $^{1}$, \\ HAIHONG DENG ${ }^{1}$, XUEJUN LI $^{1}$ and XINYUE TANG ${ }^{2}$ \\ ${ }^{1}$ Thoracic Surgeons Department; ${ }^{2}$ Oncology No. 2 Department, Guangdong Second Provincial General Hospital, \\ Guangzhou, Guangdong 510317, P.R. China
}

Received December 9, 2015; Accepted May 11, 2017

DOI: $10.3892 / \mathrm{ol} .2017 .7457$

\begin{abstract}
Bufadienolides are a type of cardiotonic steroids isolated from the skin and parotid venom glands of the toad Bufo bufo gargarizans Cantor, and exhibit wide-spectrum anticancer activities. However, the effects and mechanisms of bufadienolides on esophageal squamous cell carcinoma (ESCC) cells remain unknown. In the present study, the anticancer activities of two bufadienolides, bufotalin and bufalin, were examined in vitro and in vivo. The results demonstrated that bufotalin and bufalin effectively inhibited the viability of ESCC cells, with half-maximal inhibitory concentration $\left(\mathrm{IC}_{50}\right.$ ) values of 0.8-3.6 $\mu \mathrm{M}$. However, bufotalin and bufalin exhibited lower toxicity towards Het-1A human esophageal squamous cells, indicating their high selectivity towards cancer cells. Mechanistic studies revealed that bufotalin effectively induced ESCC cell apoptosis, as characterized by DNA fragmentation and nuclear condensation, which was primarily mediated through activation of caspase family members. In addition, treatment of ESCC cells with bufotalin markedly activated tumor protein p53 (p53) phosphorylation. Transfection of cells with p53 small interfering RNA markedly inhibited bufotalin-induced p53 phosphorylation and significantly inhibited bufotalin-induced cell apoptosis. Furthermore, bufotalin demonstrated in vivo anticancer efficacy in a tumor-bearing nude mice model, where bufotalin effectively inhibited Eca-109 xenograft tumor growth in a time- and dose-dependent manner, through activation of the p53 signaling pathway. Collectively, the results from the present study suggested that bufadienolides exert anticancer effects against ESCC by regulating the p53 signaling pathway.
\end{abstract}

Correspondence to: Dr Junhong Lv, Thoracic Surgeons Department, Guangdong Second Provincial General Hospital, 466 Xingang Road, Building 7, No. 3 Compound, Guangzhou, Guangdong 510317, P.R. China

E-mail: junhonglv@163.com

Key words: bufadienolides, apoptosis, esophageal squamous cell carcinoma, tumor protein p53

\section{Introduction}

Cancer, a prevalent health concern, is a complex disease that affects the quality of life and life expectancy of patients globally (1). The growth of transformed cells is regulated by a number of genes that also regulate normal cell proliferation (2). At present, chemotherapy is one of the most important modalities for cancer treatments; however, it is not completely effective (3). Drug discovery has identified a number of natural products that may be able to treat cancer (4). The skin of amphibians fulfills a wide range of functions necessary for their survival and previous studies have demonstrated that amphibians, including frogs and toads, possess a number of bioactive agents in their skin $(5,6)$. This has led to a growing interest in whether these agents may exhibit anticancer effects.

The extracts of amphibian skin have been used in Chinese medicine to treat different types of diseases $(7,8)$. A number of natural products have been identified as major pharmacological constituents in toad skin, including hydrosoluble indole alkaloids (bufotenine, bufotenidine, and cinobufotenine) and liposoluble steroidal cardiac glycosides, including bufadienolides $(9,10)$. Bufadienolides, including cinobufagin, bufotalin and bufalin, have been demonstrated to inhibit $\mathrm{Na}^{+} \mathrm{K}^{+}$-ATPase and exhibit wide-spectrum cytotoxic effects on a number of human cancer cells (11). Wen et al (12) demonstrated the anti-inflammatory and anti-nociceptive activities of bufalin in rodents. In addition, a previous study demonstrated that cinobufacini effectively induced the apoptosis of lens epithelial cells by suppressing the expression of B-cell lymphoma 2 (Bcl-2) family proteins (13). Due to its low toxicity, the medication prevented and treated posterior capsule opacification (14). In addition, bufalin induced cancer cell apoptosis by repressing the expression of microRNA-181a to decrease the gene expression of Bcl-2 (15). Wang et al (16) demonstrated that bufalin and cinobufagin induced HepG2 cell apoptosis through the extrinsic Fas- and intrinsic mitochondria-mediated signaling pathways, where the Fas-mediated caspase-10-dependent pathway may serve a more important function (16). Bufalin inhibited SK-Hep1 cell migration and invasion by inhibiting the nuclear factor- $\kappa$-light-chain-enhancer of activated B cells and matrix metalloproteinase-2/-9- pathways (17). Cinobufacini induced the apoptosis of T-47D cells in a caspase-3-dependent manner (18). In pancreatic cancer cells, 
bufalin exhibited its anticancer action through induction of cell cycle arrest and cell apoptosis (19). Arenobufagin has been demonstrated to induce human hepatocellular carcinoma cell apoptosis and autophagy by inhibiting the phosphoinositide 3-kinase/RAC- $\alpha$ serine/threonine-protein kinase/mammalian target of rapamycin signaling pathway (20). These results have demonstrated the application potential of bufadienolides for the treatment of cancer; however, the effects and the underlying molecular mechanisms of the effect of bufadienolides on esophageal squamous cell carcinoma (ESCC) cells remain elusive

In the present study, the anticancer activities of two bufadienolides, bufotalin and bufalin, were investigated in vitro and in vivo. The results suggested that bufotalin and bufalin effectively impeded the viability of a panel of five ESCC cell lines by inducing cell apoptosis through activation of the tumor protein p53 (p53) signaling pathway. Furthermore, bufotalin exhibited in vivo anticancer efficacy in a nude mouse model, where bufotalin inhibited the growth of tumors through activation of the p53 signaling pathway. Overall, these findings indicated that bufadienolides exert their effects against ESCC through the regulation of the p53 signaling pathway.

\section{Materials and methods}

Reagents and materials. Bufotalin (10102631) and bufalin (11070631) standards were provided by Shanghai Tauto Biotech Co., Ltd. (Shanghai, China). All other reagents were purchased from Sigma-Aldrich; Merck KGaA (Darmstadt, Germany).

Cell culture. Human ESCC cell lines (Eca-109, EC9706, TE5, Hec2 and TE11) were purchased from the American Type Culture Collection (Manassas, VA, USA) and the non-malignant human esophageal squamous cell line Het-1A was purchased from GuangZhou Jennio Biotech Co., Ltd. (Guangzhou, China). Eca-109, EC9706, TE5, Hec2 and TE11 cells were cultured and maintained in RPMI-1640 (Hyclone; GE Healthcare Life Sciences, Logan, UT, USA) supplemented with $10 \%$ fetal bovine serum (FBS; Gibco; Thermo Fisher Scientific, Inc., Waltham, MA, USA), penicillin (100 U/ml) and streptomycin $(50 \mu \mathrm{g} / \mathrm{ml})$ at $37^{\circ} \mathrm{C}$ in a humidified incubator with $5 \% \mathrm{CO}_{2}$. Het-1A cells were cultured and maintained in Dulbecco's modified Eagle's medium (DMEM; Hyclone; GE Healthcare Life Sciences) supplemented with $10 \%$ FBS, penicillin $(100 \mathrm{U} / \mathrm{ml})$ and streptomycin $(50 \mu \mathrm{g} / \mathrm{ml})$ at $37^{\circ} \mathrm{C}$ in a humidified incubator with $5 \% \mathrm{CO}_{2}$.

Examination of cell viability by MTT assay. The effects of bufotalin on cell viability were determined using an MTT assay as previously described (10). Briefly, Eca-109 $\left(2 \times 10^{4}\right.$ cells $\left./ \mathrm{ml}\right)$, EC9706 $\left(2 \times 10^{4}\right.$ cells $\left./ \mathrm{ml}\right)$, TE5 $\left(2 \times 10^{4}\right.$ cells $\left./ \mathrm{ml}\right)$, Hec2 $\left(2 \times 10^{4}\right.$ cells $\left./ \mathrm{ml}\right)$, TE11 $\left(2 \times 10^{4}\right.$ cells $\left./ \mathrm{ml}\right)$ and Het $1 \mathrm{~A}$ cells $\left(2 \times 10^{4}\right.$ cells $\left./ \mathrm{ml}\right)$ were cultured in DMEM supplemented with $10 \% \mathrm{FBS}, 100 \mathrm{U} / \mathrm{ml}$ penicillin and $50 \mu \mathrm{g} / \mathrm{ml}$ streptomycin at $37^{\circ} \mathrm{C}$ in a humidified incubator under $5 \% \mathrm{CO}_{2}$. The cell viability $\left(2 \times 10^{4}\right.$ cells $\left./ \mathrm{ml}\right)$ following treatment with 2 and $4 \mu \mathrm{M}$ bufalin or bufotalin at $37^{\circ} \mathrm{C}$ for $72 \mathrm{~h}$ was determined by the MTT assay. Eca-109, EC9706, TE5, Hec2, TE11 and Het-1A cells without drug treatment functioned as control groups.
The color intensity was measured at $575 \mathrm{~nm}$ on a microplate spectrophotometer (VersaMax; Molecular Devices, LLC, Sunnyvale, CA, USA) (21).

Flow cytometry analysis. Following exposure to different treatments as aforementioned in the MTT assay section, Eca-109, EC9706, TE5, Hec2, TE11 and Het-1A cells $\left(2 \times 10^{4}\right.$ cells $\left./ \mathrm{ml}\right)$ were washed with PBS twice and then fixed with $70 \%$ ethanol at $-20^{\circ} \mathrm{C}$ in the dark overnight. The fixed cells were stained with propidium iodide $(50 \mu \mathrm{g} / \mathrm{ml})$ for $2 \mathrm{~h}$ at $37^{\circ} \mathrm{C}$ in the dark and then flow cytometry was performed using a Coulter ${ }^{\circledR}$ Epics ${ }^{\circledR} \mathrm{XL}^{\mathrm{TM}}$ flow cytometer (Beckman Coulter, Inc., Brea, CA, USA). Apoptotic cells were quantified using the sub-G1 peak in the cell cycle distribution histogram as previously described (22).

Examination of apoptotic DNA fragmentation by terminal deoxynucleotidyl transferase dUTP nick end labeling (TUNEL) and DAPI co-staining assay. Following treatment, cells $\left(1 \times 10^{5}\right.$ cells $\left./ \mathrm{ml}\right)$ cultured in confocal dishes were fixed with formaldehyde for $10 \mathrm{~min}$ at room temperature and permeabilized with $0.1 \%$ Triton X-100 in PBS for 2 min at $4^{\circ} \mathrm{C}$. The cells were then incubated with $100 \mu \mathrm{l} /$ well TUNEL reaction mixture containing nucleotide mixture and terminal deoxynucleotidyl transferase for $1 \mathrm{~h}$ at $37^{\circ} \mathrm{C}$ and then stained with DAPI $(1 \mu \mathrm{g} / \mathrm{ml})$ for $20 \mathrm{~min}$ at $37^{\circ} \mathrm{C}$. Following washing with PBS, the cells were examined on a confocal fluorescence microscope (Zeiss LSM 510 Meta confocal microscope; Zeiss AG, Oberkochen, Germany) as described previously (10). Three fields of view were captured and selected randomly.

Activation of caspases by bufadienolides. The enzymatic activities of caspase-3, -8 and -9 in cells treated with bufadienolides were determined with a fluorometric method using specific caspase substrates (Ac-DEVD-AMC for caspase-3, Ac-IETD-AMC for caspase-8 and Ac-LEHD-AMC for caspase-9 respectively), as described previously (16).

Western blot analysis. Following treatment with 2 and $4 \mu \mathrm{M}$ bufotalin for $24 \mathrm{~h}$, cells $\left(1 \times 10^{5}\right.$ cells $\left./ \mathrm{ml}\right)$ were harvested using a cell scraper at $4^{\circ} \mathrm{C}$ and the cell pellets were collected by centrifugation at a speed of $1,000 \mathrm{xg}$ for $15 \mathrm{~min}$ at $4^{\circ} \mathrm{C}$, and were lysed in RIPA cell lysis buffer on ice for $1 \mathrm{~h}$. The total proteins were obtained from supernatant after centrifugation $(16,099 \times \mathrm{g}$ ) at $4^{\circ} \mathrm{C}$ for $30 \mathrm{~min}$. The protein concentration of cell lysates was determined using a BCA assay kit (Sigma-Aldrich; Merck $\mathrm{KGaA}$ ) as described previously (23). Equal amounts of protein (20 $\mu \mathrm{g}$ /lane) were separated on a 12\% SDS-PAGE gel and transferred to polyvinylidene fluoride membranes. Following blocking with $5 \%$ non-fat milk at $4^{\circ} \mathrm{C}$ for $2 \mathrm{~h}$, the membranes were incubated with primary antibodies p53 (cat. no. 9282; Cell Signaling Technology, Inc., Danvers, MA, USA) and phosphorylated p53 serine-15 (p-p53; cat. no. 9284, Cell Signaling Technology, Inc.) both at a dilution of 1:1,000 at $4^{\circ} \mathrm{C}$ overnight. Following washing with TBS solution, the membranes were incubated with corresponding secondary antibodies for $2 \mathrm{~h}$ at $4^{\circ} \mathrm{C}$ and visualized by Pierce enhanced chemiluminescence western blotting substrate (Pierce; Thermo Fisher Scientific, Inc., Waltham, MA, USA). 
p53 small interfering (si)RNA transfection. Cells were seeded at a density of $2 \times 10^{6}$ cells $/ \mathrm{ml}$ in 6 -well plates, which were allowed to grow to $60 \%$ confluence after $24 \mathrm{~h}$. The cells were then incubated with p53 siRNA (100 nmol/l; cat. no. 6231; Cell Signaling Technology, Inc.) in serum-free DMEM for 24 h, with Lipofectamine ${ }^{\circledR} 2000$ (cat. no. 11668027; Thermo Fisher Scientific, Inc.) used as a transfection reagent according to the manufacturer's protocol. Then, fresh serum-free DMEM medium with $10 \%$ fetal bovine serum was added to each well for $24 \mathrm{~h}$ with or without 2 and $4 \mu \mathrm{M}$ bufadienolides at $4^{\circ} \mathrm{C}$ for $24 \mathrm{~h}$. Control cells were transfected with a fluorescein-labeled non-targeted scramble control siRNA (cat. no. 78193; Cell Signaling Technology, Inc.), which allowed for the monitoring of transfection efficiency. Following siRNA transfection, the cells were subjected to apoptotic analysis as aforementioned.

Immunofluorescence analysis of protein expression in cells. Levels of p53 (cat. no. 9282; Cell Signaling Technology, Inc.) and phosphorylated p53 serine-15 (p-p53; cat. no. 9284; Cell Signaling Technology, Inc.), both at a dilution of 1:1,000 in the treated cells were analyzed by immunofluorescence. Following treatment, $4 \times 10^{4}$ cells were seeded, washed with PBS and fixed with $3.7 \%$ formaldehyde in PBS for $15 \mathrm{~min}$ at $4^{\circ} \mathrm{C}$. Following rinsing with PBS, the cells were permeabilized with $0.2 \%$ Triton $\mathrm{X}-100$ for $5 \mathrm{~min}$ at room temperature. The permeabilized cells were then blocked for $30 \mathrm{~min}$ with $0.1 \%$ bovine serum albumin (Gibco; Thermo Fisher Scientific, Inc.) at room temperature and incubated with p-p53 primary antibodies at $4^{\circ} \mathrm{C}$ overnight. Cells were then incubated with Alexa-488 labeled anti-rabbit immunoglobulin $\mathrm{G}$ (IgG) antibody (cat. no. 4412; 1:250; Cell Signaling Technology, Inc.) for $1 \mathrm{~h}$ at room temperature. The cells nuclei were stained with Hoechst $33342(1 \mu \mathrm{g} / \mathrm{ml})$ for $20 \mathrm{~min}$ at $37^{\circ} \mathrm{C}$. A total of 3 fields of view of each slide were selected randomly and analyzed using a confocal fluorescence microscope (Zeiss LSM 510 Meta confocal microscope with LSM 510 software; Zeiss AG).

Tumor xenograft in nude mice. The female Balb/c nude mice (age, 3-4 weeks; weight, 14-15 g; n=40) were purchased from Beijing HuaFuKang Bioscience Co., Ltd. (Beijing, China) and housed ad libitum at $\sim 25^{\circ} \mathrm{C}$. The pressure difference was $25 \mathrm{~Pa}$, relative humidity was $40-70 \%$, and the light/dark cycle was $12 / 12 \mathrm{~h}$. The mice were acclimated to the environment for 10 days prior to treatment. Eca-109 cells $\left(5 \times 10^{6}\right)$ suspended in PBS were injected subcutaneously into the right lower hind flank of each 6-week-old male nude mouse. The mice were then randomly assigned into three groups with 10 mice in each group. After 10 days, bufotalin dissolved in solution (N.N-di methylformamide ${ }_{\mathrm{v}}$ :Tween-80 : $_{\mathrm{v}}$ :line $_{\mathrm{v}}=10: 2: 88$ ) was administered (intraperitoneal; 2 or $4 \mathrm{mg} / \mathrm{kg}$ body weight every other day) for 20 days. Control mice received an equal volume of the vehicle (saline). The body weights and tumor volumes were monitored every two days. After 20 days, tumor size had grown to $\sim 10 \mathrm{~mm}$ (the humane end-point) and tumor xenografts were collected and weighed.

The tumor dimension was measured with calipers, and the tumor volume was calculated with the following formula: Volume $=1 \mathrm{x} \mathrm{w}^{2} / 2$, where 1 is the maximal length and $\mathrm{w}$ is the width. A portion of the tumors from control and treated groups were fixed in $10 \%$ formalin at room temperature for $24 \mathrm{~h}$, dehydrated with graded ethanol (80, 90, 95 and 100\%) and embedded in paraffin (section thickness, $4 \mu \mathrm{m}$ ) for hematoxylin $(0.5 \mathrm{mg} / \mathrm{ml})$ staining for $15 \mathrm{~min}$ at room temperature and eosin $(0.5 \mathrm{mg} / \mathrm{ml})$ staining for $5 \mathrm{~min}$ at room temperature and immunohistochemical analyses. Immunofluorescence staining analysis (magnification, x20) using a confocal fluorescence microscope (Zeiss LSM 510 Meta confocal microscope with LSM 510 software; Zeiss AG) was performed with anti-p-p53 (dilution, 1:100; Ser ${ }^{15}$; cat. no. 9284; Cell Signaling Technology, Inc.) at $4{ }^{\circ} \mathrm{C}$ overnight and ki67 antibodies at $4{ }^{\circ} \mathrm{C}$ overnight (dilution, 1:100; cat. no. 9129; Cell Signaling Technology, Inc.) and Alexa Fluor 488-conjugated anti-rabbit IgG (cat. no. 4340; Cell Signaling Technology, Inc.) antibodies (1:100) at room temperature for $1 \mathrm{~h}$. Blocking was performed with $5 \%$ bovine serum albumin for $15 \mathrm{~min}$ at room temperature. All animal studies were approved by the Animal Experimentation Ethics Committee of Guangdong No. 2 Provincial People's Hospital (Guanzhou, China).

Statistical analysis. In the present study, all experiments were performed using SPSS 10.0 (SPSS, Inc., Chicago, IL, USA) $\geq 3$ times and the data were presented as the mean \pm standard error of the mean. Differences between two groups were analyzed using a two-tailed Student's t-test and differences among three or more groups was analyzed by one-way analysis of variance and Fisher's least significant difference post hoc test. $\mathrm{P}<0.05$ was considered to indicate a statistically significant difference. Quantitative analysis was performed using Quantity-one 1D software (Bio-Rad Laboratories, Inc., Hercules, CA, USA).

\section{Results and Discussion}

Bufotalin and bufalin effectively inhibit ESCC cell viability. The in vitro anticancer effects of bufotalin and bufalin (chemical structures are illustrated in Fig. 1A) were investigated in five human ESCC cancer cell lines, including Eca-109, EC9706, TE5, Hec2 and TE11, and a human esophageal squamous cell line Het-1A. Following treatment with $2 \mu \mathrm{M}$ bufotalin and $4 \mu \mathrm{M}$ bufalin at $37^{\circ} \mathrm{C}$ for $72 \mathrm{~h}$, cell viability was detected using an MTT assay. Half-maximal inhibitory concentration $\left(\mathrm{IC}_{50}\right)$ values revealed that bufotalin and bufalin effectively inhibited the cell viability of ESCC cells, particularly bufotalin, which exhibited $\mathrm{IC}_{50}$ values of $0.8,1.2,3.2$, 2.4 and 3.6 $\mu \mathrm{M}$ for Eca-109, TE5, EC9706, Hec2 and TE11 cells, respectively (Fig. 1B). This improved effect of bufotalin compared with bufalin was observed in the TE5, EC9706 and HET-1A cell lines. Notably, bufotalin and bufalin exhibited markedly lower toxicity towards Het-1A human esophageal squamous cells, indicating the high selectivity of these two compounds towards cancer cells. Collectively, these results indicated that bufotalin and bufalin may have applications in the treatment of ESCC.

Activation of cell apoptosis by bufotalin. Dysregulation of cell apoptosis (also known as programmed cell death) has been demonstrated to be associated with a number of human chronic diseases, including cancer (24). Induction of cancer cell apoptosis has been identified as an effective way to treat cancer (25). The growth inhibitory activities of the majority 

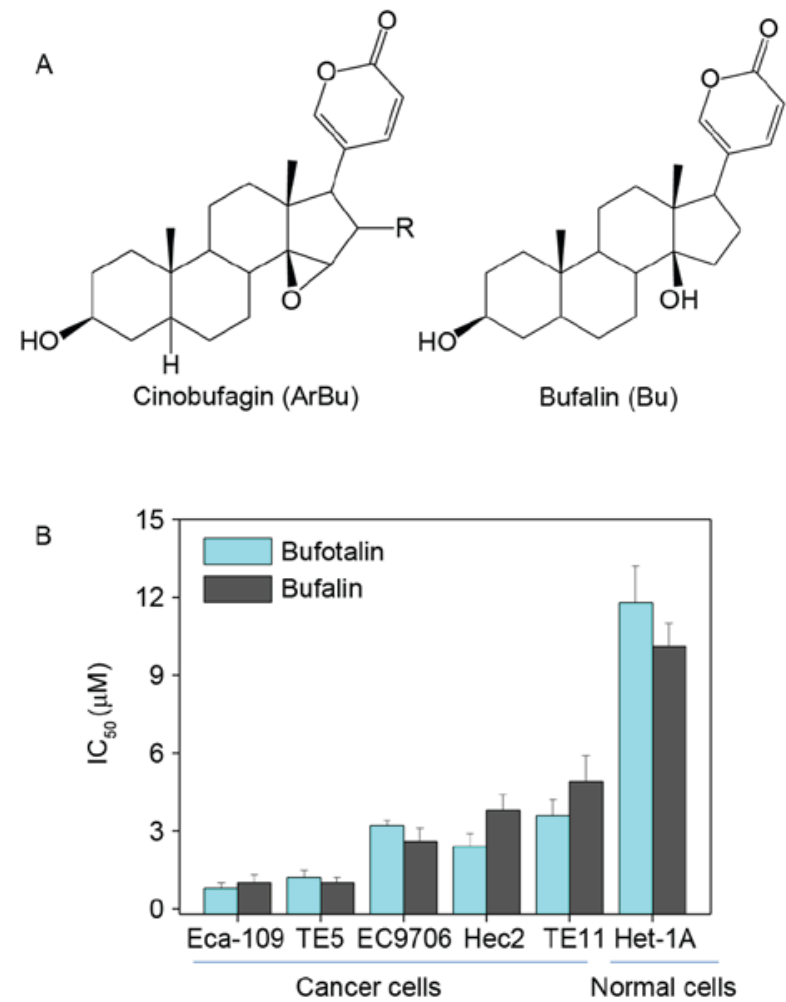

Figure 1. In vitro anticancer activities of $2 \mu \mathrm{M}$ bufotalin and $4 \mu \mathrm{M}$ bufalin. (A) Chemical structure of bufotalin and bufalin. (B) Cytotoxicity of bufotalin and bufalin towards human cancer and normal cells. The ESCC cells and Het-1A human esophageal squamous cells were treated with bufotalin and bufalin for $72 \mathrm{~h}$, and the cell viability was examined by MTT assay.

of anticancer drugs on cancer cells are achieved through the induction of apoptosis (26). Results from the MTT assay in the present study demonstrated that bufotalin exhibited markedly increased anticancer efficacy compared with bufalin. Therefore, further studies were performed to explore the molecular mechanisms underlying the effects of bufotalin on cancer cell death. As demonstrated by a TUNEL-DAPI co-staining assay, Eca-109 cells exposed to 2 and $4 \mu \mathrm{M}$ bufotalin for $24 \mathrm{~h}$ exhibited marked and dose-dependent apoptosis compared with the control (Fig. 2). In addition, DAPI staining revealed that Eca-109 cells treated with bufotalin exhibited DNA fragmentation and nuclear condensation. Quantitative analysis (data not shown) by counting the apoptotic cells, revealed that bufotalin induced dose-dependent increased apoptosis in the treated cells compared with the control cells.

Caspase family proteins act as important regulators in the induction of apoptosis through the enzymolysis of series of substrates (27). Activated caspases subsequently induce proteolytic cleavage of poly ADP ribose polymerase and finally result in cell apoptosis (27). Cell apoptosis is initiated by two central mechanisms; the death receptor-mediated extrinsic and the mitochondria-mediated intrinsic apoptotic pathways (28). In the present study, intracellular caspase activities in cells exposed to bufotalin were measured in order to examine the requirement of caspase proteins for bufotalin-induced apoptosis. Exposure of Eca-109 cells to bufotalin significantly induced the activation of caspase-3, -8 and -9 compared with the control (caspase-3, $\mathrm{P}<0.01$ and $\mathrm{P}<0.01$; caspase- $8, \mathrm{P}<0.05$ and $\mathrm{P}<0.01$; caspase-9,
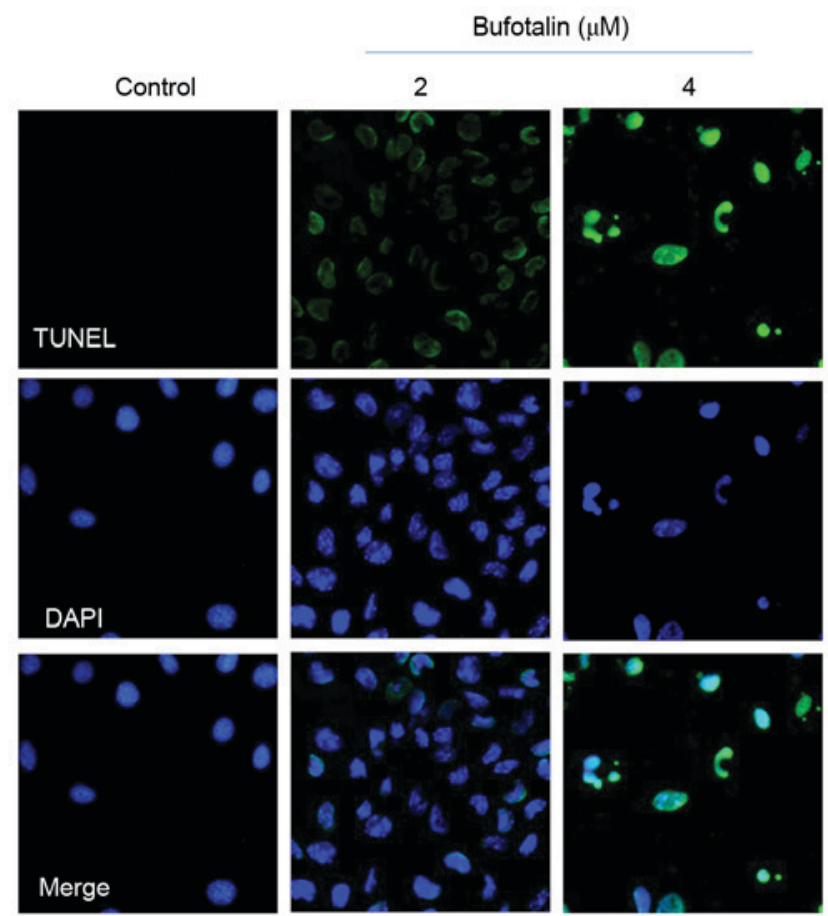

Figure 2. Apoptotic DNA fragmentation and nuclear condensation induced by bufotalin, examined using a TUNEL and DAPI co-staining assay. The cells were treated with bufotalin for $24 \mathrm{~h}$. Green fluorescence indicates apoptotic DNA fragmentation, while the arrows in the DAPI-staining images indicate the nuclear condensation. Values at the bottom of the images indicate the percentage of apoptotic cells. Magnification, x200. TUNEL, terminal deoxynucleotidyl transferase dUTP nick end labeling.

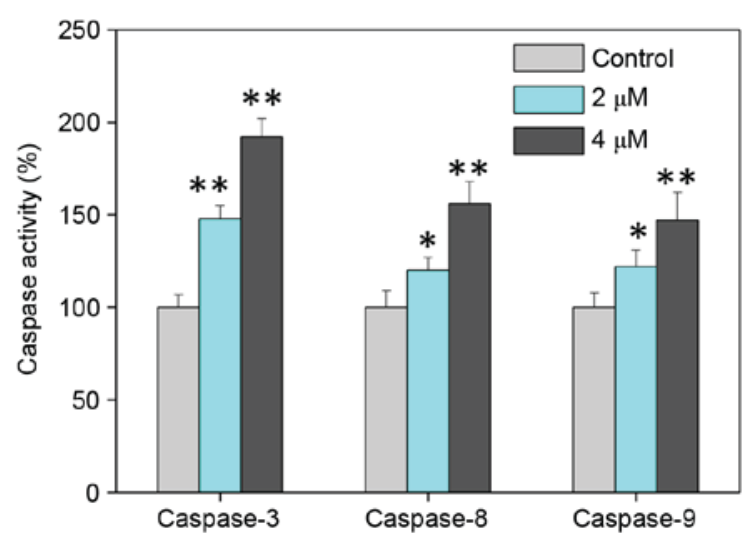

Figure 3. Caspase activities of esophageal squamous cell carcinoma cells exposed to bufotalin as examined using specific fluorogenic substrates. The cells were treated with bufotalin and bufalin for $24 \mathrm{~h}$. ${ }^{*} \mathrm{P}<0.05$ and ${ }^{* *} \mathrm{P}<0.01$ vs. control.

$\mathrm{P}<0.05$ and $\mathrm{P}<0.01$ for 2 and $4 \mu \mathrm{M}$ bufotalin, respectively; Fig. 3). The activation of caspase-9 suggested the induction of the mitochondria-mediated apoptotic pathway, while the activation of caspase- 8 indicated the induction of death receptor-mediated apoptosis (29). These results revealed that bufotalin induces ESCC cell apoptosis through the activation of caspase-mediated apoptosis, with the involvement of mitochondria and death receptors.

Involvement of p53 in bufotalin-induced cell apoptosis. The p53 tumor suppressor integrates a number of stress signals 
A

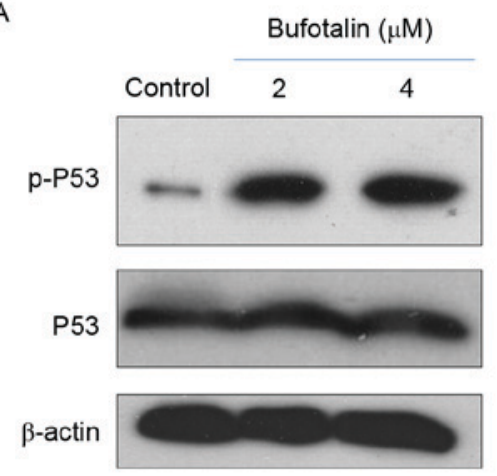

B

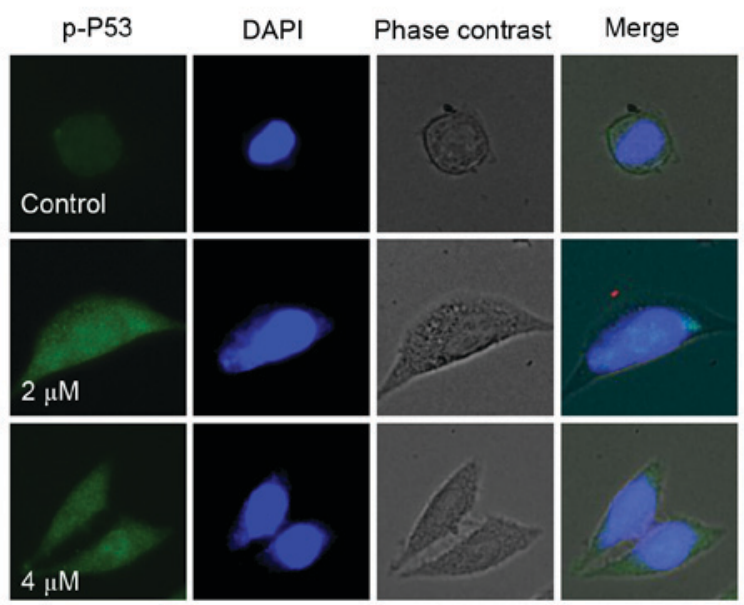

Figure 4. Activation of 533 in bufotalin-induced apoptosis. (A) Phosphorylation of p53 at Ser15 and expression of total p53 in Eca-109 cells treated with bufotalin at 2 and $4 \mu \mathrm{M}$ for $24 \mathrm{~h}$, assessed by western blotting. Quantitative analysis was performed using Quantity-one 1D software. (B) Immunofluorescence of p-p53 in cells treated with bufotalin for 24 h. p53, tumor protein p53; p-, phosphorylated.

and signaling pathways (30). One of the most important functions of p53 is the regulation of cell apoptosis by activation of the expression of p53-inducible targets, including Bcl-2 associated $\mathrm{X}$ protein, p53 upregulated modulator of apoptosis and cyclin-dependent kinase inhibitor 1 in cancer cells, which may promote tumor chemotherapy by enhancing the apoptosis-inducing activities of anticancer drugs $(25,31)$. In addition, p53 regulates cell apoptosis through upregulation of the expression of DNA damage-associated proteins and the inhibition of DNA repair (32). Therefore, in the present study, the levels of total p53 and p-p53 were examined by western blot analysis and fluorescence imaging in order to elucidate the molecular mechanisms underlying the anticancer effects of bufotalin. Bufotalin markedly increased the expression of p53 total protein and p-p53 (Ser15) in Eca-109 cells compared with the control (Fig. 4A). Fluorescence imaging confirmed the increase in p-p53 levels following treatment with bufotalin (Fig. 4B). The involvement of p53 in bufotalin-induced ESCC cell apoptosis was further verified using RNA interference. Transfection with p53 siRNA markedly inhibited bufotalin-induced p53 phosphorylation compared with bufotalin treatment alone (Fig. 5A) and significantly decreased apoptotic cell death compared with bufotalin treatment alone $(\mathrm{P}<0.05$; Fig. 5B), whereas transfection with the negative
A

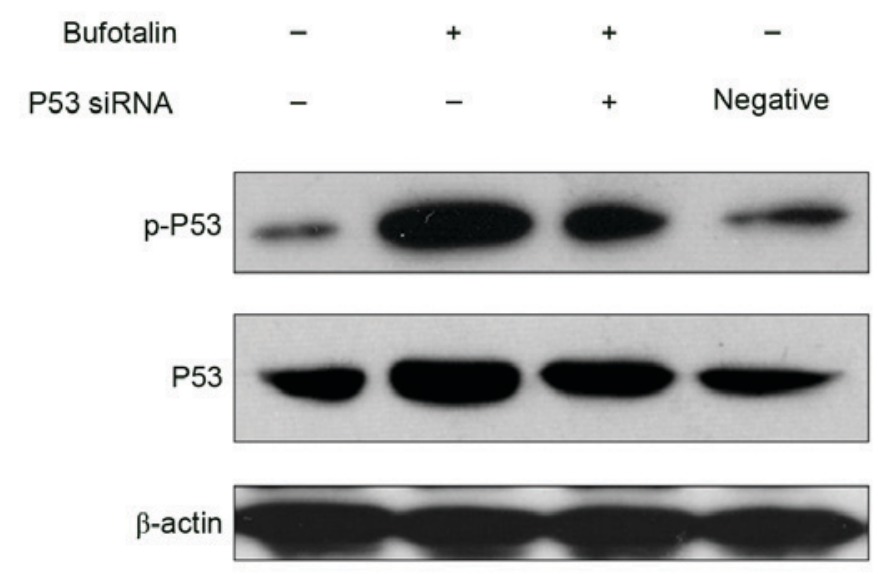

B

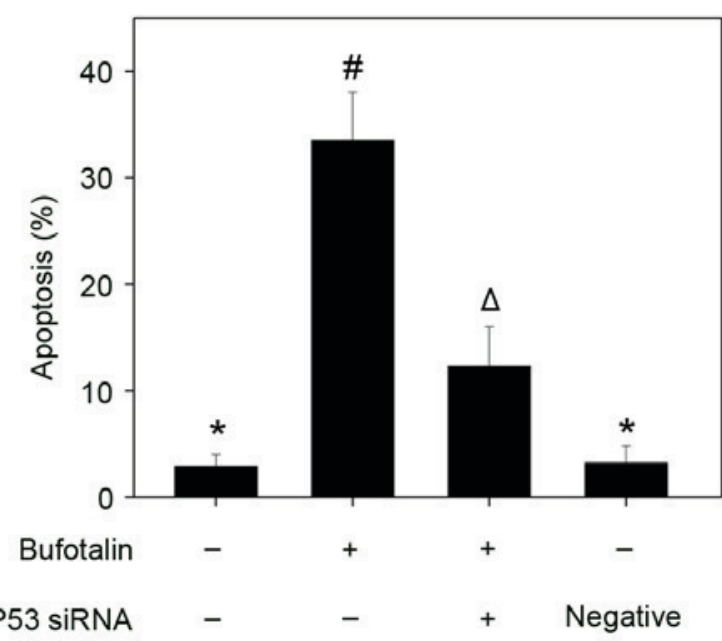

Figure 5. Involvement of p53 in bufotalin-induced apoptosis. (A) Western blot analysis of p53 and p-p53 expression following transfection with p53 siRNA and (B) the effects on cell apoptosis following treatment with bufotalin $(4 \mu \mathrm{M} ; 24 \mathrm{~h})$, as examined by propidium iodide flow cytometry analysis. Results were obtained from three independent experiments. Bars with different symbols $\left(",{ }^{*}\right.$ and $\left.{ }^{\Delta}\right)$ are statistically different from every other group at $\mathrm{P}<0.05$, and the groups with the same symbol * (control group and negative group) indicate no significant difference at $\mathrm{P}<0.05$. Control siRNA was used as negative control, and no bufotalin was added in this group. p53, tumor protein p53; p-, phosphorylated; siRNA, small interfering RNA.

control siRNA exhibited no significant effects (Fig. 5A and B). These results suggested that bufotalin activates ESCC cell apoptosis through activation of the p53-dependent signaling pathway.

In vivo anticancer activities of bufotalin with involvement of p53 phosphorylation. The in vivo anticancer activity of bufotalin against ESCC was evaluated in Eca-109 xenografts in a nude mice model. Following administration of 2 or $4 \mathrm{mg} / \mathrm{kg}$ body weight bufotalin every other day for 20 days, the tumor weight was significantly reduced from 2.3 to 1.4 and $1.0 \mathrm{~g}$, respectively $(\mathrm{P}<0.05$ and $\mathrm{P}<0.01$, respectively; Fig. $6 \mathrm{~A})$. In addition, the tumor volume was markedly decreased to 68 and $46 \%$ of the control group (Fig. 6B). The mice that received bufotalin treatment maintained normal body weight throughout the treatment process. These results demonstrate the in vivo anticancer efficacy of bufotalin against ESCC. In addition, 
A

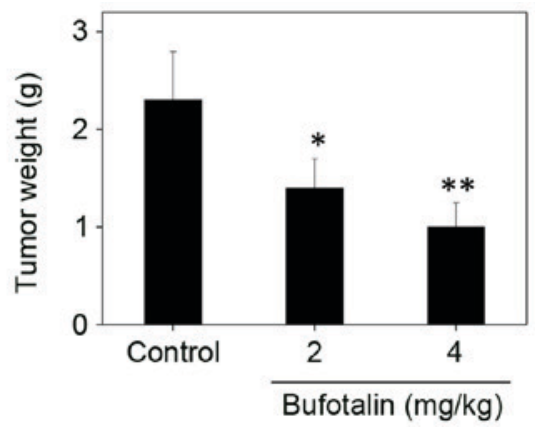

B

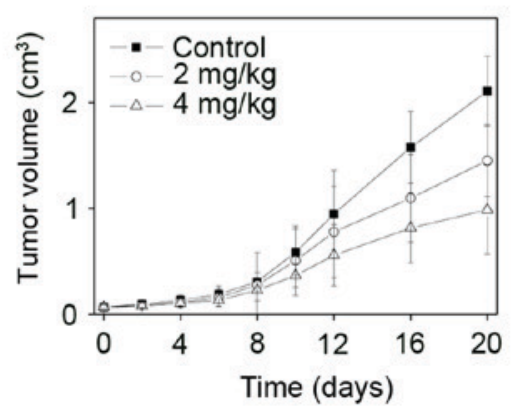

C

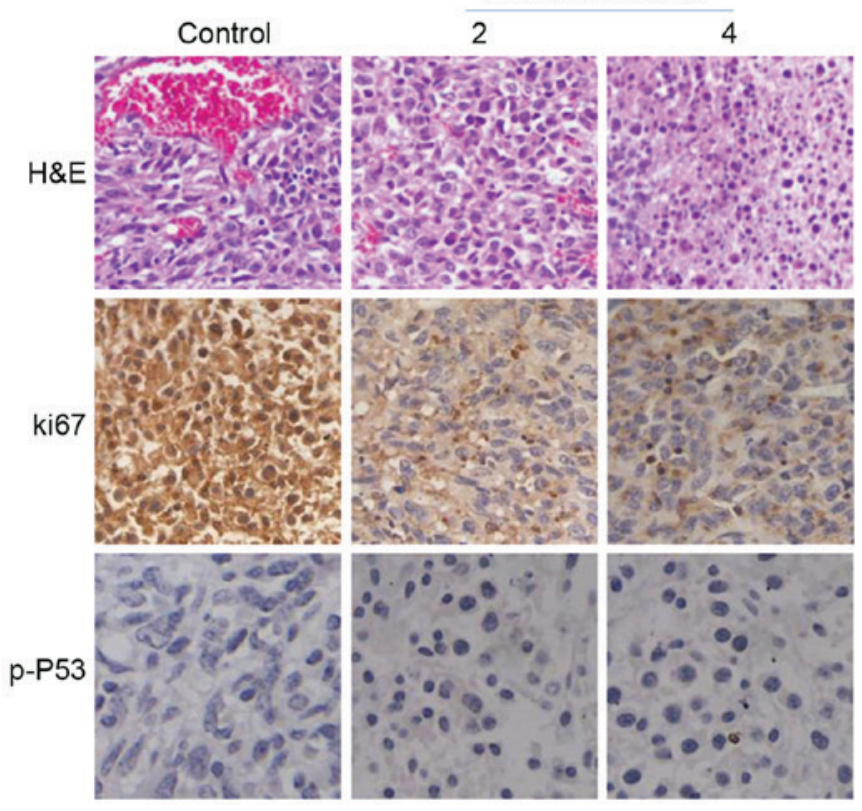

Figure 6. In vivo antitumor efficacy of bufotalin. (A) Xenograft tumor weight and (B) volume following 20 days of treatment with bufotalin. BALB/c nude mice bearing Eca-109 xenograft tumors were treated with either a vehicle ( $0.1 \%$ dimethyl sulfoxide solvent control) or bufotalin ( 2 or $4 \mathrm{mg} / \mathrm{kg}$ every other day). (C) H\&E staining and immunohistochemical analysis of the expression levels of Ki-67 and p-p53 in Eca-109 xenograft tumors. "P<0.05 and ${ }^{* *} \mathrm{P}<0.01$ vs. the control. $\mathrm{H} \& \mathrm{E}$, hematoxylin and eosin; $\mathrm{p} 53$, tumor protein p53; p-, phosphorylated.

immunostaining of histological sections was performed in order to examine the in vivo anticancer mechanisms. A marked decrease in blood vessel and cancer cell density was observed in tumors treated with 2 and $4 \mathrm{mg} / \mathrm{kg}$ bufotalin compared with the control (Fig. 6C). The expression of Ki-67, a biomarker of proliferation, was markedly inhibited by bufotalin. In addition, the expression of phosphorylated p53 was markedly enhanced following treatment. These in vivo data support the hypothesis that bufotalin inhibits tumor growth through activation of the p53 signaling pathway.

To conclude, in the present study, the in vitro and in vivo anticancer efficacies of two bufadienolides (bufotalin and bufalin) were examined. The results demonstrated that bufotalin and bufalin effectively suppressed the viability of ESCC cell lines through induction of cell apoptosis occurring through activation of the p53 signaling pathway. In addition, bufotalin demonstrated in vivo anticancer efficacy in a nude mouse model, where bufotalin markedly suppressed tumor growth through activation of the p53 signaling pathway. Collectively, these results illustrated the therapeutic potential of bufadienolides against ESCC by regulating the p53 signaling pathway.

\section{Acknowledgements}

The present study was supported by the Construction of Scientific Medicine Research Project of Guangdong Province (grant no. 2013 20131105).

\section{References}

1. Song G, Cheng L, Chao Y, Yang K and Liu Z: Emerging nanotechnology and advanced materials for cancer radiation therapy. Adv Mater 29, 2017.

2. Humbert PO, Verona R, Trimarchi JM, Rogers C, Dandapani S and Lees JA: E2f3 is critical for normal cellular proliferation. Genes Dev 14: 690-703, 2000.

3. Chang Y, He L, Li Z, Zeng L, Song Z, Li P, Chan L, You Y, Yu XF, Chu PK and Chen T: Designing core-shell gold and selenium nanocomposites for cancer radiochemotherapy. Acs Nano 11: 4848-4858, 2017.

4. Singh SK, Banerjee S, Acosta EP, Lillard JW and Singh R: Resveratrol induces cell cycle arrest and apoptosis with docetaxel in prostate cancer cells via a p53/p21(WAF1/CIP1) and p27(KIP1) pathway. Oncotarget 8: 17216-17228, 2017.

5. Liong M, Lu J, Kovochich M,Xia T, Ruehm SG, Nel AE, Tamanoi F and Zink JI: Multifunctional inorganic nanoparticles for imaging, targeting, and drug delivery. ACS Nano 2: 889-896, 2008.

6. Oelkrug C, Hartke M and Schubert A: Mode of action of anticancer peptides (ACPs) from amphibian origin. Anticancer Res 35: 635-643, 2015.

7. Chen T and Wong YS: Selenocystine induces apoptosis of A375 human melanoma cells by activating ROS-mediated mitochondrial pathway and p53 phosphorylation. Cell Mol Life Sci 65: 2763-2775, 2008 .

8. Chen T and Wong YS: Selenocystine induces caspase-independent apoptosis in MCF-7 human breast carcinoma cells with involvement of $\mathrm{p} 53$ phosphorylation and reactive oxygen species generation. Int J Biochem Cell Biol 41: 666-676, 2009.

9. Qi FH, Li AY, Lv H, Zhao L, Li JJ, Gao B and Tang W: Apoptosis-inducing effect of cinobufacini, Bufo bufo gargarizans Cantor skin extract, on human hepatoma cell line BEL-7402. Drug Discov Ther 2: 339-343, 2008.

10. Chen Y, Qiao L, Yu B, Li G, Liu C, Ji L and Chao H: Mitochondria-specific phosphorescent imaging and tracking in living cells with an AIPE-active iridium(III) complex. Chem Commun (Camb) 49: 11095-11097, 2013.

11. Wang Z, Wen J, Zhang J, Ye M and Guo D: Simultaneous determination of four bufadienolides in human liver by high-performance liquid chromatography. Biomed Chromatogr 18: 318-322, 2004.

12. Wen L, Huang Y, Xie X, Huang W, Yin J, Lin W, Jia Q and Zeng W: Anti-inflammatory and antinociceptive activities of bufalin in rodents. Mediators Inflamm 2014: 171839, 2014.

13. Wang DL, Qi FH, Xu HL, Inagaki Y, Orihara Y, Sekimizu K, Kokudo N, Wang FS and Tang W: Apoptosis-inducing activity of compounds screened and characterized from cinobufacini by bioassay-guided isolation. Mol Med Rep 3: 717-22, 2010.

14. Xu GX and Wang TT: Apoptosis of lens epithelial cells induced by cinobufagin in vitro. Int J Ophthalmol 3: 128-131, 2010. 
15. Zhai XF, Fang FF, Liu Q, Meng YB, Guo YY and Chen Z: MiR-181a contributes to bufalin-induced apoptosis in PC-3 prostate cancer cells. BMC Complement Altern Med 13: 325, 2013.

16. Wang F, Zhang D, Zhang Q, Chen Y, Zheng D, Hao L, Duan C, Jia L, Liu G and Liu Y: Synergistic effect of folate-mediated targeting and verapamil-mediated P-gp inhibition with paclitaxel-polymer micelles to overcome multi-drug resistance. Biomaterials 32 : 9444-9456, 2011

17. Chen YY, Lu HF, Hsu SC, Kuo CL, Chang SJ, Lin JJ, Wu PP, Liu JY, Lee CH, Chung JG and Chang JB: Bufalin inhibits migration and invasion in human hepatocellular carcinoma SK-Hep1 cells through the inhibitions of NF-kB and matrix metalloproteinase-2/-9-signaling pathways. Environ Toxicol 30 74-82, 2015.

18. Wang W, Shi A and Fan Z: Apoptosis of T-47D cells induced by cinobufacini via a caspase-3-dependent manner. Chem Res Chinese Univer 30: 108-113, 2014.

19. Li M, Yu X, Guo H, Sun L, Wang A, Liu Q, Wang X and Li J: Bufalin exerts antitumor effects by inducing cell cycle arrest and triggering apoptosis in pancreatic cancer cells. Tumour Biol 35 2461-2471, 2014

20. Zhang DM, Liu JS, Deng LJ, Chen MF, Yiu A, Cao HH, Tian HY, Fung KP, Kurihara H, Pan JX and Ye WC: Arenobufagin, a natural bufadienolide from toad venom, induces apoptosis and autophagy in human hepatocellular carcinoma cells through inhibition of PI3K/Akt/mTOR pathway. Carcinogenesis 34: 1331-1342, 2013

21. Luo H, Wang F, Bai Y, Chen T and Zheng W: Selenium nanoparticles inhibit the growth of HeLa and MDA-MB-231 cells through induction of $\mathrm{S}$ phase arrest. Colloids Surf B Biointerfaces 94 304-308, 2012

22. Yang F, Tang Q, Zhong X, Bai Y, Chen T, Zhang Y, Li Y and Zheng W: Surface decoration by Spirulina polysaccharide enhances the cellular uptake and anticancer efficacy of selenium nanoparticles. Int J Nanomedicine 7: 835-844, 2012.

23. Zhang Y, Li X, Huang Z, Zheng W, Fan C and Chen T: Enhancement of cell permeabilization apoptosis-inducing activity of selenium nanoparticles by ATP surface decoration. Nanomedicine 9: 74-84, 2013.
24. Maiese K, Chong ZZ, Shang YC and Wang S: Targeting disease through novel pathways of apoptosis and autophagy. Expert Opin Ther Targets 16: 1203-1214, 2012.

25. Chen T and Wong YS: Selenocystine induces apoptosis of A375 human melanoma cells by activating ROS-mediated mitochondrial pathway and p53 phosphorylation. Cell Mol Life Sci 65: 2763-2775, 2008.

26. Chen T and Wong YS: Selenocystine induces caspaseindependent apoptosis in MCF-7 human breast carcinoma cells with involvement of p53 phosphorylation and reactive oxygen species generation. Int J Biochem Cell Biol 41: 666-676, 2009.

27. Riedl SJ and Shi Y: Molecular mechanisms of caspase regulation during apoptosis. Nat Rev Mol Cell Biol 5: 897-907, 2004.

28. He L, Lai H and Chen T: Dual-function nanosystem for synergetic cancer chemo-/radiotherapy through ROS-mediated signaling pathways. Biomaterials 51: 30-42, 2015.

29. Li M, Lai L, Zhao Z and Chen T: Aquation is a crucial activation step for anticancer action of ruthenium(II) polypyridyl complexes to trigger cancer cell apoptosis. Chem Asian J 11: 310-320, 2016.

30. Xie Q, Lan G, Zhou Y, Huang J, Liang Y, Zheng W, Fu X, Fan C and Chen T: Strategy to enhance the anticancer efficacy of X-ray radiotherapy in melanoma cells by platinum complexes, the role of ROS-mediated signaling pathways. Cancer Lett 354: 58-67, 2014.

31. Fridman JS and Lowe SW: Control of apoptosis by p53. Oncogene 22: 9030-9040, 2003.

32. He L, Huang Y, Zhu H, Pang G, Zheng W, Wong YS and Chen T: Cancer-targeted monodisperse mesoporous silica nanoparticles as carrier of ruthenium polypyridyl complexes to enhance theranostic effects. Adv Funct Mater 24: 2754-2763, 2014.

This work is licensed under a Creative Commons Attribution-NonCommercial-NoDerivatives 4.0 International (CC BY-NC-ND 4.0) License. 